\title{
Magic sizes of cationic and protonated argon clusters
}

\author{
Michael Gatchell, ${ }^{1,2, *}$ Paul Martini, ${ }^{1}$ Lorenz Kranabetter, ${ }^{1}$ Bilal Rasul, ${ }^{1,3}$ and Paul Scheier ${ }^{1}$ \\ ${ }^{1}$ Institut für Ionenphysik und Angewandte Physik, Universität Innsbruck, Technikerstr. 25, A-6020 Innsbruck, Austria \\ ${ }^{2}$ Department of Physics, Stockholm University, 10691 Stockholm, Sweden \\ ${ }^{3}$ Department of Physics, University of Sargodha, 40100 Sargodha, Pakistan
}

(Received 29 March 2018; published 29 August 2018)

\begin{abstract}
There has long been a discrepancy between the size distributions of $\mathrm{Ar}_{n}{ }^{+}$clusters measured by different groups regarding whether or not magic numbers appear at sizes corresponding to the closure of icosahedral (sub-)shells. We show that the previously observed magic cluster size distributions are likely the result of an unresolved $\mathrm{Ar}_{n} \mathrm{H}^{+}$ component, i.e., from protonated argon clusters. We find that the proton impurity gives cluster geometries that are much closer to those for neutral rare-gas clusters, which are known to form icosahedral structures, than for the pure cationic clusters, explaining why the mass spectra from protonated argon clusters better matches these structural models. Our results thus show that even small impurities, e.g., a single proton, can significantly influence the properties of clusters.
\end{abstract}

DOI: 10.1103/PhysRevA.98.022519

\section{INTRODUCTION}

Rare-gas clusters are some of the simplest chemical systems studied, with many of their structural properties deduced from basic sphere packing models [1] or classical two-body interactions such as the Lennard-Jones 6-12 potential $[2,3]$. Theoretical studies have shown that the global energy minima of such clusters containing less than a few hundred particles (with few exceptions [4]) prefer icosahedral geometries where shell closures (and the filling of faces on the polyhedra) are associated with enhanced stabilities [5-7].

It is alas difficult to experimentally study the structures and stabilities of neutral rare-gas clusters [8]. Charged clusters are, however, easily studied using mass spectrometric techniques. In 1981, Echt et al. reported that clusters of xenon formed by supersonic expansion and ionized by electron impact showed enhanced abundances at clusters sizes of 13, 19, 23, 25, 55, 71, $81,87,101,135$, and 147 , which could be explained by sphere packing in the formation of icosahedral structures [1]. This was followed by numerous studies showing similar magic cluster size series in $\mathrm{He}_{n}{ }^{+}[9,10], \mathrm{Ar}_{n}{ }^{+}[11,12], \mathrm{Ne}_{n}{ }^{+}$[13], and $\mathrm{Kr}_{n}{ }^{+}$ $[14,15]$ clusters.

Early on it was noted that these series of magic cluster sizes were not always reproducible. This is particularly true in the case of $\mathrm{Ar}_{n}{ }^{+}$, where experimental results can be placed in one of two categories: (1) where increased abundances of $\mathrm{Ar}_{n}{ }^{+}$ clusters with $n=13,19,23,26,29,32,34,43,46,49,55,64$, 71,81 , and 87 are observed [11,12], and (2) where the strongest anomaly of $\mathrm{Ar}_{n}{ }^{+}$cluster sizes below $n=81$ is a particularly low abundance of $\mathrm{Ar}_{20}{ }^{+}$[15-20], which is considered to be antimagic. The reasons for the observed differences have been

\footnotetext{
*michael.gatchell@uibk.ac.at
}

Published by the American Physical Society under the terms of the Creative Commons Attribution 4.0 International license. Further distribution of this work must maintain attribution to the author(s) and the published article's title, journal citation, and DOI. debated for more than 30 years, mainly revolving around how the clusters are formed (e.g., whether the clusters are born neutral before being ionized, or are grown around a charged core), and remains poorly understood [21].

In this paper we present high-resolution mass spectrometry measurements of pure $\mathrm{Ar}_{n}{ }^{+}$clusters and protonated $\mathrm{Ar}_{n} \mathrm{H}^{+}$ clusters that can be separated up to cluster sizes of $n \approx 100$. We find that the pure $\mathrm{Ar}_{n}{ }^{+}$cluster series show few abundance anomalies (in agreement with results from Refs. [15-20]), while the protonated $\mathrm{Ar}_{n} \mathrm{H}^{+}$clusters show pronounced magic numbers in agreement with the results on $\mathrm{Ar}_{n}{ }^{+}$clusters by Harris et al. [11,12] and neutral Lennard-Jones clusters [5]. We thus come to believe that the significant differences observed in past studies of argon clusters is due to contributions from protonated clusters. This conclusion is further motivated by $a b$ initio calculations of pure and protonated argon clusters.

\section{EXPERIMENTAL DETAILS}

We produce the argon clusters in He nanodroplets using the setup described in detail in Refs. [22-24]. Briefly, droplets of $\mathrm{He}$ containing on average a few million atoms are formed by the supersonic expansion of compressed (2.5 MPa) He through a nozzle that is cooled to $9.5 \mathrm{~K}$. The droplets capture $\mathrm{Ar}$ and $\mathrm{H}_{2}$ gas that is injected in a pickup chamber, which condense into clusters in the superfluid $0.37 \mathrm{~K}$ droplets. The droplets are ionized by impact of $76 \mathrm{eV}$ electrons and the positively charged products are analyzed with a reflectron time-of-flight mass spectrometer (Tofwerk AG model HTOF). The mass spectra are calibrated and analyzed using the ISOTOPEFIT software [25]. This method of producing rare-gas clusters has been used in the past to study $\mathrm{Ar}_{n}{ }^{+}$[20] and $\mathrm{Kr}_{n}{ }^{+}$[22] clusters, giving results is good agreement with other techniques [15-19].

\section{RESULTS AND DISCUSSION}

In Fig. 1 we show an overview spectrum from He nanodroplets doped with $\mathrm{Ar}$ and $\mathrm{H}_{2}$ prior to ionization. At low 


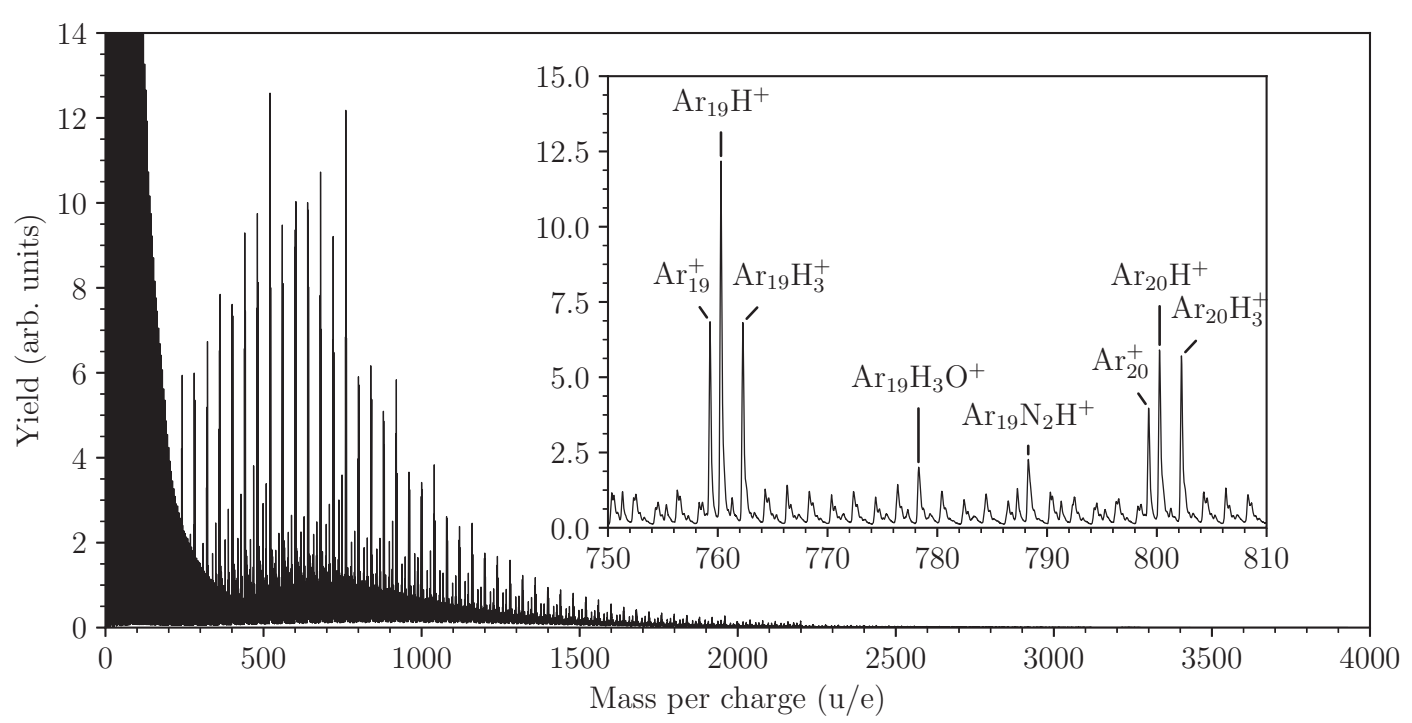

FIG. 1. Mass spectrum of positively charged products from $\mathrm{He}$ nanodroplets doped with $\mathrm{Ar}$ and $\mathrm{H}_{2}$ that are ionized by $76 \mathrm{eV}$ electrons. The distribution at low masses is the pure $\mathrm{He}_{n}{ }^{+}$series and at higher masses clusters of $\mathrm{Ar}_{n}{ }^{+}, \mathrm{Ar}_{n} \mathrm{H}^{+}$, and $\mathrm{Ar}_{n} \mathrm{H}_{3}{ }^{+}$dominate. The inset shows a zoomed-in region of the spectrum where the particularly strong $\mathrm{Ar}_{19} \mathrm{H}^{+}$peak is apparent.

masses (below about $400 \mathrm{u} / e$ ) we mainly see the contribution from pure $\mathrm{He}_{n}{ }^{+}$clusters from larger neutral droplets that fragment upon ionization. At higher masses the spectrum is dominated by $\mathrm{Ar}_{n}{ }^{+}, \mathrm{Ar}_{n} \mathrm{H}^{+}$, and $\mathrm{Ar}_{n} \mathrm{H}_{3}{ }^{+}$clusters that are free of helium. The inset of Fig. 1 shows a zoom-in of the mass range covering $\operatorname{Ar}_{19} X^{+}$and $\operatorname{Ar}_{20} X^{+}$systems. With the resolution of the mass spectrometer $(R \approx 4000)$ we can clearly separate the individual cluster series, the relative intensities of which can be tuned by varying the $\mathrm{Ar}$ and $\mathrm{H}_{2}$ pressures in the pickup region.

Size distributions of $\mathrm{Ar}_{n}{ }^{+}, \mathrm{Ar}_{n} \mathrm{H}^{+}$, and $\mathrm{Ar}_{n} \mathrm{H}_{3}{ }^{+}$clusters are shown in Fig. 2 from separate measurements where we have optimized the intensities of each series. This does not affect the specific structures in each series, but can shift the underlying

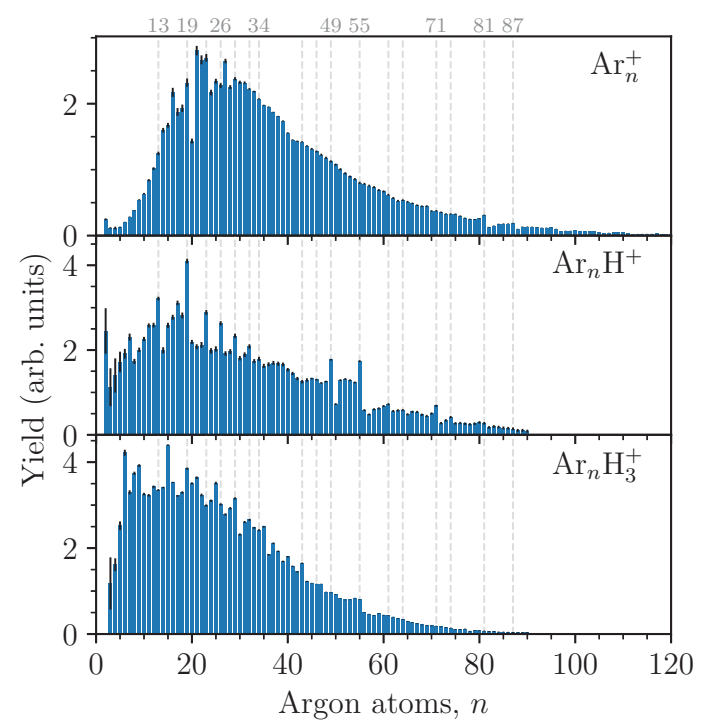

FIG. 2. Cluster size distributions of $\mathrm{Ar}_{n}{ }^{+}, \mathrm{Ar}_{n} \mathrm{H}^{+}$, and $\mathrm{Ar}_{n} \mathrm{H}_{3}{ }^{+}$. Dashed lines show the magic cluster sizes reported by Harris et al. $[11,12]$ for $\mathrm{Ar}_{n}{ }^{+}$. The statistical uncertainties are indicated by black error bars. log-normal distributions that result from the pickup statistics. Vertical dashed lines in each panel show the magic cluster sizes reported by Harris et al. [11,12] for pure $\mathrm{Ar}_{n}{ }^{+}$clusters and the most prominent of these features are labeled above the top panel. The pure $\mathrm{Ar}_{n}{ }^{+}$series (top panel of Fig. 2) displays a log-normal size distribution with few anomalies. The depleted $\mathrm{Ar}_{20}{ }^{+}$channel reported numerous times in the past [15-20] is clearly visible, as are a few other anomalies. There is a clear drop-off in intensity between $\mathrm{Ar}_{23}{ }^{+}$and $\mathrm{Ar}_{24}{ }^{+}$, one that has been reported before [20], as well as what could be interpreted as magic peaks from $\mathrm{Ar}_{16}{ }^{+}$and $\mathrm{Ar}_{27}{ }^{+}$. Although small on an absolute scale due to the underlying cluster size distribution, there are also clear abundance anomalies visible for $\mathrm{Ar}_{81}{ }^{+}$and $\mathrm{Ar}_{87}{ }^{+}$, which match magic numbers previously reported for $\mathrm{Ar}_{n}{ }^{+}$[11,12], $\mathrm{Kr}_{n}{ }^{+}$[14], and $\mathrm{Xe}_{n}{ }^{+}$[1] clusters.

The distribution of protonated Ar clusters (middle panel of Fig. 2) is clearly different from that of the pure argon clusters (top panel). It is immediately clear that every single magic size identified by Harris et al. [11] for pure $\mathrm{Ar}_{n}{ }^{+}$clusters is associated with an abundance anomaly in our $\mathrm{Ar}_{n} \mathrm{H}^{+}$series. In addition to this, there are several more subtle features that agree between the two works, such as the particularly low abundance of $\mathrm{Ar}_{50} \mathrm{H}^{+}$clusters that is followed by a plateau of relatively abundant $\mathrm{Ar}_{51} \mathrm{H}^{+}$through $\mathrm{Ar}_{54} \mathrm{H}^{+}$peaks. The main standout feature is that we also identify a magic $\mathrm{Ar}_{7} \mathrm{H}^{+}$peak, which lies below the lower limit of most $\mathrm{Ar}_{n}{ }^{+}$mass spectra found in the literature and is rarely discussed.

In the bottom panel of Fig. 2 we show a size distribution of $\mathrm{Ar}_{n} \mathrm{H}_{3}{ }^{+}$clusters. Some anomalies match the magic sizes seen with $\mathrm{Ar}_{n} \mathrm{H}^{+}$clusters (e.g., $\left.n=19,29,43,55\right)$, although most do not. It is thus clear that the specific positions of abundance anomalies, i.e., magic numbers, is indeed dependent on the types of impurities present in the argon clusters. For the remainder of this paper we will mainly focus on the $\mathrm{Ar}_{n}{ }^{+}$and $\mathrm{Ar}_{n} \mathrm{H}^{+}$clusters.

Clusters of the form $\operatorname{Ar}_{n} X^{+}$, where $X$ is some impurity atom or molecule, have been studied in the past [20,26-29], 


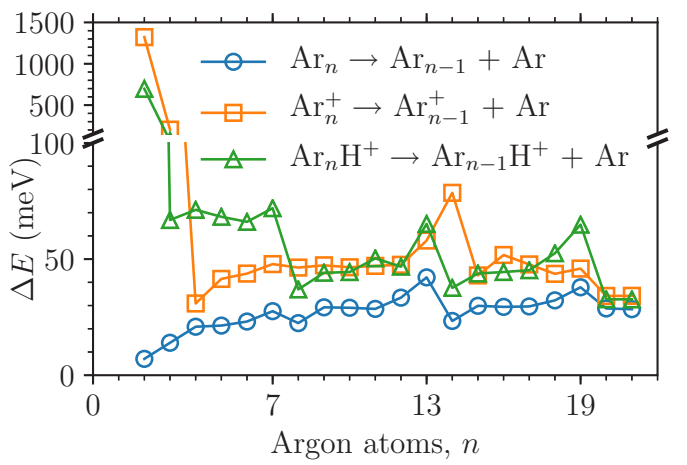

FIG. 3. Dissociation energy for losing a single Ar atom from different neutral and cationic clusters calculated at MP2(Full)/def2SVPP level. The energies included zero-point corrections but not basis-set superposition error corrections.

displaying magic features similar to those reported for pure $\mathrm{Ar}_{n}{ }^{+}$clusters. For example, a magic $\mathrm{Ar}_{54} \mathrm{~N}_{2}{ }^{+}$cluster has been identified where one of the $\mathrm{Ar}$ atoms in an icosahedral geometry is replaced with a $\mathrm{N}_{2}$ molecule [20]. Protonated argon has also been well studied for small systems such as the $\mathrm{ArH}^{+}$dimer [30,31] and the linear $\mathrm{ArHAr}^{+}$system [30,32]. However, previous experimental studies on protonated argon clusters have only investigated small clusters containing less than ten $\mathrm{Ar}$ atoms [26,29], while theory has covered $\mathrm{Ar}_{n} \mathrm{H}^{+}$ sizes up to $n=35[28,29,33]$.

To better understand our experimental results, we have performed $a b$ initio structure calculations of neutral $\mathrm{Ar}_{n}$ clusters, cationic $\mathrm{Ar}_{n}{ }^{+}$clusters, and protonated $\mathrm{Ar}_{n} \mathrm{H}^{+}$clusters for sizes up to $n=21$. The calculations were performed at MP2(Full)/def2-SVPP level using GAUSSIAN 16 [34]. This method was selected based on previous theoretical studies $[28,29]$, test calculations on the geometries of small cluster sizes, and due to the favorable scaling that allows us to study clusters with up to relatively large sizes. The evaporation energy for losing a single, neutral Ar atom as a function of cluster size of these systems is shown in Fig. 3 and the optimized structures of $\mathrm{Ar}_{13}, \mathrm{Ar}_{13} \mathrm{H}^{+}, \mathrm{Ar}_{13}{ }^{+}, \mathrm{Ar}_{14}, \mathrm{Ar}_{14} \mathrm{H}^{+}$, and $\mathrm{Ar}_{14}{ }^{+}$in Fig. 4. The geometry optimizations were carried out starting from the structures of neutral Lennard-Jones clusters [5] and in the case of the pure $\mathrm{Ar}_{n}{ }^{+}$clusters, geometries from Ref. [35] were also tested. The atomic coordinates for the lowest energy structure of each cluster is given in the Supplemental Material [36].

In Fig. 3 we can see that the curves for $\operatorname{Ar}_{n}$ clusters (blue circles) and $\mathrm{Ar}_{n} \mathrm{H}^{+}$clusters (green triangles) show the same main features, i.e., relatively tightly bound systems with $n=7,13,19$ followed by weaker systems for $n=8,14,20$. The main difference is that the protonated clusters are more tightly bound due to the presence of the charge that attracts the surrounding argon atoms to the $\mathrm{ArHAr}^{+}$unit that forms the core of the cluster (see Fig. 4). Our calculations agree well with the structure determined in previous theoretical studies of protonated [28,29,33] and cationic [35] argon clusters where sizes overlap and they readily explain the first few magic numbers observed in our experiments.

The purely cationic clusters (orange squares in Fig. 3), on the other hand, show a very different behavior. We find no

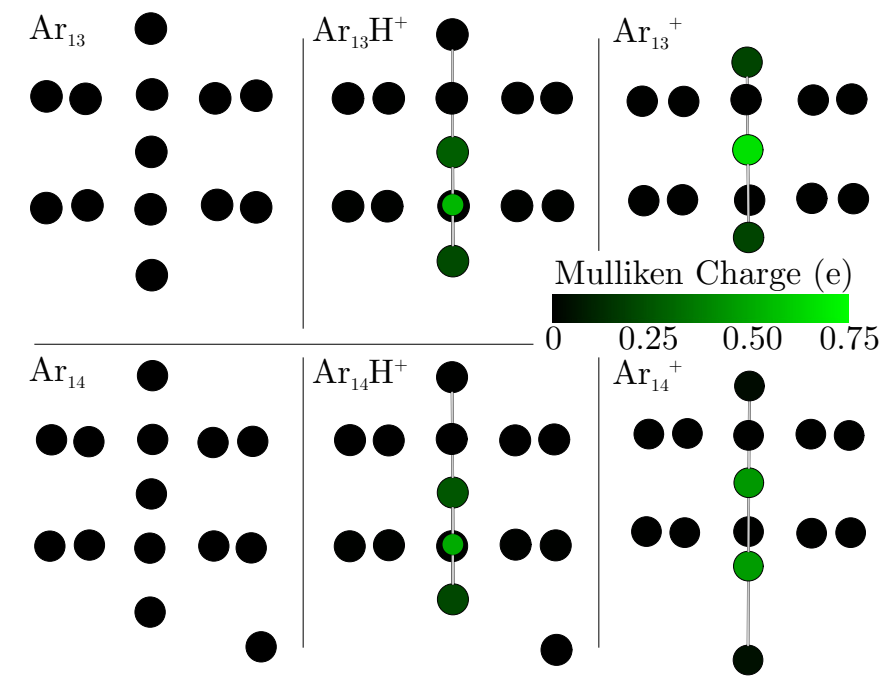

FIG. 4. Two-dimensional projections of icosahedral structures of $\mathrm{Ar}_{13}, \mathrm{Ar}_{13} \mathrm{H}^{+}$, and $\mathrm{Ar}_{13}{ }^{+}$, and structures of $\mathrm{Ar}_{14}, \mathrm{Ar}_{14} \mathrm{H}^{+}$, and $\mathrm{Ar}_{14}{ }^{+}$ optimized at MP2(Full)/def2-SVPP level. Atoms are colored based on their Mulliken charge and the proton in the center column is identified by a circle with a smaller radius than the rest. Coordinates of all calculated cluster geometries are given in the Supplemental Material.

step in binding energy after $n=7$ and the first local maxima is instead located at $n=14$. The reason for the difference in this curve compared to the other two is due to the existence of an $\mathrm{Ar}_{3}{ }^{+}$or $\mathrm{Ar}_{4}{ }^{+}$core that these clusters form around. The linear $\mathrm{Ar}_{3}{ }^{+}$core in $\mathrm{Ar}_{13}{ }^{+}$is significantly contracted compared to other Ar-Ar distances as seen in Fig. 4. This strains the icosahedral geometry so that when a 14th Ar atom is added it interacts relatively strongly with this core, forming the basis for the $\mathrm{Ar}_{4}{ }^{+}$system that is present in larger cluster sizes [35]. The larger $\mathrm{Ar}_{4}{ }^{+}$core gives the surrounding neutral $\mathrm{Ar}$ atoms more locations to closely interact with the charge center, leading to more possible competing isomers for a given cluster size. This could explain the poorer agreement between the theory and experiments for the $\mathrm{Ar}_{n}$ cluster distribution compared to the other systems. The optimal geometries of the protonated clusters, on the other hand, are very similar to the neutral systems, with the proton slotting in between two Ar atoms without significantly altering the distance between them (see Fig. 4). These compact structures are well explained by icosahedral geometries, giving the magic number series that is observed in the experiments.

The fact that the protonated argon clusters essentially retain the geometries of the neutral clusters is why the magic numbers predicted by sphere packing models are so well reproduced in the $\mathrm{Ar}_{n} \mathrm{H}^{+}$series. The pure $\mathrm{Ar}_{n}^{+}$clusters instead behave as packed spheres with a structural defect (e.g., $\mathrm{Ar}_{3}{ }^{+}$) at their core [19]. However, as the cluster sizes increase, the overall effect of this distortion on the entire cluster will decrease. This is likely the reason why abundance anomalies matching sphere packing models [1] begin to appear in the $\mathrm{Ar}_{n}{ }^{+}$mass spectrum (Fig. 2) for $n \geqslant 81$.

In light of the present results we have reevaluated data from previous studies of $\mathrm{Kr}_{n}{ }^{+}$clusters [22] performed with the same setup as the current work. While that study did identify magic cluster sizes [22], we do not find any evidence 
that protonated clusters played a roll in those results. We suspect this is because for heavier rare-gas clusters, the effect the charge has on the core of the clusters decreases, thus putting less strain on icosahedral packing of atoms. We thus do not believe that protonation plays an important role in the magic series of $\mathrm{Kr}_{n}{ }^{+}[14,15]$ and $\mathrm{Xe}_{n}{ }^{+}$[1] clusters. It does, however, seem likely that for the lighter rare gases (Ne and $\mathrm{He}$ ) protonation can have a strong effect on the geometries of charged clusters. Test calculations that we have performed on $\mathrm{Ne}_{n} \mathrm{H}^{+}$clusters show a similar behavior as we see for argon clusters, i.e., that protonated clusters better match the structures of neutral clusters. Isotopic mixtures of $\mathrm{Ne}$ atoms would make the experimental distinction between protonated and pure Ne clusters more difficult (this is not a problem for the nearly isotopically pure Ar), but we are nonetheless currently performing measurements on these systems with our setup.

\section{CONCLUSION}

We have shown that protonated argon clusters show very different characteristics than pure, cationic argon clusters. The protonated clusters display magic sizes that perfectly match the magic cluster series reported by Harris et al. [11,12] for pure $\mathrm{Ar}_{n}{ }^{+}$clusters, indicating that their measurements may have contained an unresolved contribution from protonated clusters, likely originating from some impurity (e.g., water) in their setup. This could thus solve the long-standing disagreement between different studies on argon clusters regarding the nature and origin of abundance anomalies in the mass spectra $[11,12,15-20]$. It also highlights the dramatic differences small impurities can play in the formation and characteristics of clusters and small nanoparticles, similar to what has been observed regarding the electronic properties of small carbon cluster anions and their hydrides [37], and the role that hydrogen plays in stabilizing metal clusters [38].

\section{ACKNOWLEDGMENTS}

This work was supported by the Austrian Science Fund FWF (projects P26635 and W1259) and the Swedish Research Council (Contract No. 2016-06625). The computational results presented have been achieved (in part) using the HPC infrastructure LEO of the University of Innsbruck.
[1] O. Echt, K. Sattler, and E. Recknagel, Phys. Rev. Lett. 47, 1121 (1981).

[2] T. Ikeshoji, B. Hafskjold, Y. Hashi, and Y. Kawazoe, Phys. Rev. Lett. 76, 1792 (1996).

[3] C. Rey, L. J. Gallego, M. P. Iñiguez, and J. A. Alonso, Physica B: Condens. Matter 179, 273 (1992).

[4] R. H. Leary and J. P. K. Doye, Phys. Rev. E 60, R6320(R) (1999).

[5] D. J. Wales and J. P. K. Doye, J. Phys. Chem. A 101, 5111 (1997).

[6] Y. Xiang, H. Jiang, W. Cai, and X. Shao, J. Phys. Chem. A 108, 3586 (2004).

[7] Y. Xiang, L. Cheng, W. Cai, and X. Shao, J. Phys. Chem. A 108, 9516 (2004).

[8] R. Brühl, R. Guardiola, A. Kalinin, O. Kornilov, J. Navarro, T. Savas, and J. P. Toennies, Phys. Rev. Lett. 92, 185301 (2004).

[9] P. W. Stephens and J. G. King, Phys. Rev. Lett. 51, 1538 (1983).

[10] H. Buchenau, E. L. Knuth, J. Northby, J. P. Toennies, and C. Winkler, J. Chem. Phys. 92, 6875 (1990).

[11] I. A. Harris, R. S. Kidwell, and J. A. Northby, Phys. Rev. Lett. 53, 2390 (1984).

[12] I. A. Harris, K. A. Norman, R. V. Mulkern, and J. A. Northby, Chem. Phys. Lett. 130, 316 (1986).

[13] T. D. Märk and P. Scheier, Chem. Phys. Lett. 137, 245 (1987).

[14] M. Lezius, P. Scheier, A. Stamatovic, and T. D. Märk, J. Chem. Phys. 91, 3240 (1989).

[15] W. Miehle, O. Kandler, T. Leisner, and O. Echt, J. Chem. Phys. 91, 5940 (1989).

[16] T. A. Milne and F. T. Greene, J. Chem. Phys. 47, 4095 (1967).

[17] A. Ding and J. Hesslich, Chem. Phys. Lett. 94, 54 (1983).

[18] P. Scheier and T. D. Märk, Int. J. Mass Spectrom. Ion Proces. 76, R11 (1987).

[19] N. E. Levinger, D. Ray, M. L. Alexander, and W. C. Lineberger, J. Chem. Phys. 89, 5654 (1988).

[20] F. Ferreira da Silva, P. Bartl, S. Denifl, O. Echt, T. D. Märk, and P. Scheier, Phys. Chem. Chem. Phys. 11, 9791 (2009).

[21] K. Vafayi and K. Esfarjani, J. Cluster Sci. 26, 473 (2015).

[22] H. Schöbel, P. Bartl, C. Leidlmair, S. Denifl, O. Echt, T. D. Märk, and P. Scheier, Eur. Phys. J. D 63, 209 (2011).
[23] T. Kurzthaler, B. Rasul, M. Kuhn, A. Lindinger, P. Scheier, and A. M. Ellis, J. Chem. Phys. 145, 064305 (2016).

[24] M. Kuhn, M. Renzler, J. Postler, S. Ralser, S. Spieler, M. Simpson, H. Linnartz, A. G. G. M. Tielens, J. Cami, A. Mauracher, Y. Wang, M. Alcamí, F. Martín, M. K. Beyer, R. Wester, A. Lindinger, and P. Scheier, Nat. Commun. 7, 13550 (2016).

[25] S. Ralser, J. Postler, M. Harnisch, A. M. Ellis, and P. Scheier, Int. J. Mass Spectrom. 379, 194 (2015).

[26] G. Hvistendahl, O. W. Saastad, and E. Uggerud, Int. J. Mass Spectrom. Ion Proces. 98, 167 (1990).

[27] M. Lezius, P. Scheier, and T. D. Märk, Chem. Phys. Lett. 196, 118 (1992).

[28] K. T. Giju, S. Roszak, and J. Leszczynski, J. Chem. Phys. 117, 4803 (2002).

[29] D. C. McDonald, D. T. Mauney, D. Leicht, J. H. Marks, J. A. Tan, J. L. Kuo, and M. A. Duncan, J. Chem. Phys. 145, 231101 (2016).

[30] V. E. Bondybey and G. C. Pimentel, J. Chem. Phys. 56, 3832 (1972).

[31] J. W. C. Johns, J. Mol. Spectrosc. 106, 124 (1984).

[32] H. M. Kunttu and J. A. Seetula, Chem. Phys. 189, 273 (1994).

[33] T. Ritschel, P. J. Kuntz, and L. Zülicke, Eur. Phys. J. D 33, 421 (2005).

[34] M. J. Frisch et al., Gaussian 16 rev. a.03, 2016.

[35] T. Ikegami, T. Kondow, and S. Iwata, J. Chem. Phys. 98, 3038 (1993).

[36] See Supplemental Material at http://link.aps.org/supplemental/ 10.1103/PhysRevA.98.022519 for structures of $\mathrm{Ar}_{n}, \mathrm{Ar}_{n}{ }^{+}$, and $\mathrm{Ar}_{n} \mathrm{H}^{+}$clusters.

[37] G. Ito, T. Furukawa, H. Tanuma, J. Matsumoto, H. Shiromaru, T. Majima, M. Goto, T. Azuma, and K. Hansen, Phys. Rev. Lett. 112, 183001 (2014).

[38] B. Kiran, P. Jena, X. Li, A. Grubisic, S. T. Stokes, G. F. Ganteför, K. H. Bowen, R. Burgert, and H. Schnöckel, Phys. Rev. Lett. 98, 256802 (2007). 